\title{
THE PERFORMANCE OF THE NEW TCDQ SYSTEM IN THE LHC DUMPING REGION
}

\author{
B. Goddard, A. Presland, W. Weterings, CERN, Geneva, Switzerland
}

\section{Abstract}

The superconducting quadrupole magnet Q4 and other downstream LHC machine elements risk destruction in the event of a beam dump that is not synchronised with the abort gap. To protect these elements, a single sided mobile graphite diluter block TCDQ, in combination with a two-sided secondary collimator TCS and iron shield TCDQM, will be installed in front of Q4. This protection system should also intercept spurious particles in the beam abort gap to prevent quenches from occurring during regular beam aborts, and must also intercept the particles from the secondary halo during low beam lifetime without provoking quenches. The conceptual design of the TCDQ system is briefly presented, with the load conditions and performance criteria. The FLUKA simulations are described results discussed in the context of the expected performance for LHC operation.

\section{INTRODUCTION}

The LHC beam dumping system [1] uses a fast-pulsed kicker magnet system MKD to deflect the beam horizontally into a set of septum magnets MSD. A single sided mobile diluter TCDQ, in combination with a collimator TCS and an iron shield TCDQM, will be installed in front of the Q4 magnet, Fig. 1, for protection against a beam dump not synchronised with the abort gap.

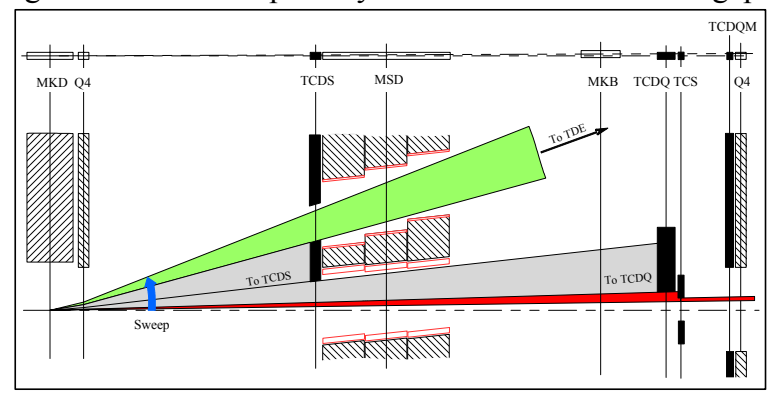

Figure 1: Schematic layout of TCDQ, TCS \& TCDQM.

The TCDQ is a $6.0 \mathrm{~m}$ single-sided graphite absorber block (density $1.7 \mathrm{~g} / \mathrm{cm}^{3}$ ), positioned $12.5 \mathrm{~m}$ in front of the Q4 magnet, followed by a short two-sided TCS collimator which will be positioned at a slightly $(\sim 1 \sigma)$ closer setting than the TCDQ proper, to intercept the bulk of the secondary halo. This provision allows a relaxation of the tolerances for the TCDQ, to avoid problems with positioning relative to the beam. A $2.1 \mathrm{~m}$ iron TCDQM mask directly in front of Q4 completes the system.

\section{PERFORMANCE CRITERIA}

The performance criteria of the TCDQ system depend on the damage and quench limits of the downstream superconducting magnets. Best estimates for the various figures of merit are collated in Table 1 [2].

Table 1: Assumed limits for superconducting elements

\begin{tabular}{|l|l|l|}
\hline Limit & $\mathbf{4 5 0} \mathbf{G e V}$ & $\mathbf{7 ~ T e V}$ \\
\hline Damage; instantaneous & $87 \mathrm{~J} / \mathrm{cm}^{3}$ & $87 \mathrm{~J} / \mathrm{cm}^{3}$ \\
\hline Quench; instantaneous & $35 \mathrm{~mJ} / \mathrm{cm}^{3}$ & $4 \mathrm{~mJ} / \mathrm{cm}^{3}$ \\
\hline Quench; localised & $1-10 \mathrm{~mW} / \mathrm{cm}^{3}$ & $0.2-5 \mathrm{~mW} / \mathrm{cm}^{3}$ \\
\hline Quench; total power & $34 \mathrm{~W}$ & $34 \mathrm{~W}$ \\
\hline
\end{tabular}

\section{Protection from asynchronous abort}

In the event of an asynchronous abort, the TCDQ will intercept $\sim 36$ proton bunches, with the transverse intensity profile as shown in Fig. 2 for $7 \mathrm{TeV}$. The energy deposited in the protection system elements, and in the MCBY and MQY, should not exceed the damage limit.

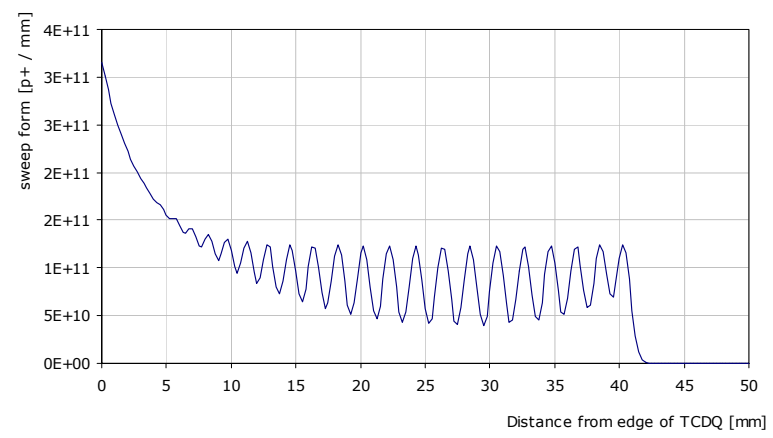

Figure 2: Asynchronous dump intensity profile at $7 \mathrm{TeV}$.

At $450 \mathrm{GeV}$ the LHC aperture is $7.5 \sigma$. The TCS is set at $7 \sigma$, the TCDQ at $8 \sigma$. The primary beam intensity should be reduced to $2 \times 10^{12} \mathrm{p}+$. This requires $2.5 \mathrm{~m}$ of graphite [2]; the $6 \mathrm{~m}$ TCDQ is thus largely sufficient.

At $7 \mathrm{TeV}$ the low- $\beta$ triplets have a transverse aperture of about $10 \sigma$ for squeezed optics. The TCS is set at $9 \sigma$, the TCDQ at $10 \sigma$. To protect the triplets, the system should reduce the intensity of the primary beam by a factor of 50,000. This requires $5 \mathrm{~m}$ of graphite [2]; again the $6 \mathrm{~m}$ TCDQ is sufficient.

\section{Quench protection from secondary beam halo}

The tight settings of the TCDQ mean that the system could intercept a significant continuous load from the secondary halo, shown for $450 \mathrm{GeV}$ in Fig. 3. The TCDQ system must protect the machine at the settings described above while not producing a quench in the Q4. The maximum and integrated power deposition in the superconducting coils should not exceed the quench limits in Table 1. For minimum lifetime a total of about $610^{11}$ 
$\mathrm{p}+/ \mathrm{s}$ are assumed lost. Considering the cleaning inefficiency in Fig. 3, the maximum number which can impact TCS/TCDQ is shown in Table 2.

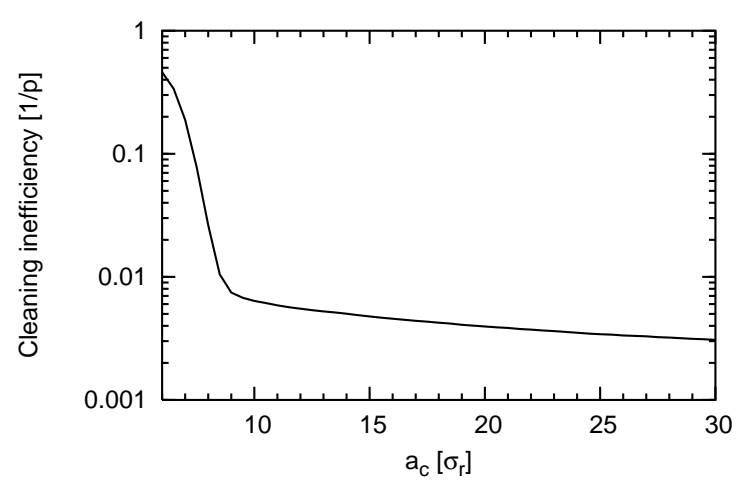

Figure 3: Secondary halo profile at $450 \mathrm{GeV}$.

Table 2: Assumed-worst case halo total beam loads

\begin{tabular}{|l|l|l|}
\hline & $\mathbf{4 5 0} \mathbf{~ G e V}$ & $\mathbf{7 ~ T e V}$ \\
\hline Load on TCS $[\mathrm{p}+/ \mathrm{s}]$ & $1.310^{11}$ & $7.510^{8}$ \\
\hline Load on TCDQ $[\mathrm{p}+/ \mathrm{s}]$ & $610^{9}$ & $4.510^{8}$ \\
\hline
\end{tabular}

During a regular beam abort, the TCDQ system will intercept particles in the abort gap. The maximum energy deposition in the superconducting Q4 coils must not exceed the instantaneous quench limit. This requirement places an upper limit on the spurious abort gap population. The impact profile is assumed to be the same as in Fig. 2 (i.e. bunched beam spread throughout the abort gap), with the total number of protons scaled to the total abort gap population. The energy deposition in the TCDQ, TCS and TCDQM must obviously not damage these protection elements.

\section{SIMULATION}

The FLUKA-2003 Monte-Carlo code [3] has been used to simulate primary and secondary particle cascades.

\section{Particle transport}

Interactions, transport and energy deposition were followed down to the kinetic energy threshold of $100 \mathrm{keV}$ for charged particles, $10 \mathrm{keV}$ for photons and $19.6 \mathrm{MeV}$ for neutrons. Particles reaching or produced below these thresholds were assumed to deposit their energy locally. Multiple Coulomb scattering was included down to the limit of Mollière's theory.

\section{Post processing}

Instantaneous temperature increases were derived from energy deposition in the adiabatic limit. A small amount of energy density $d E$ deposited in a volume $d V$ of a material with density $\rho$ causes a temperature rise $\Delta T$ determined by $\Delta T=C_{p} \rho d V d T$, where $C_{\mathrm{p}}$ is the specific heat. The dynamic specific heat $C_{\mathrm{p}}(\mathrm{T})$ for graphite and copper are given by below while that of iron is considered constant at $0.44 \mathrm{~J} / \mathrm{gK}$ over the expected range of temperature.

$$
\begin{aligned}
& C_{p}^{\text {graph }}(T)=528.75-205.9 T^{1 / 3}+154.21 T^{1 / 2}-1.53 T+9.15 \times 10^{-5} T^{2} \\
& C_{p}^{C u}(T)=381.12+0.16 T-1.09 \times 10^{-4} T^{2}
\end{aligned}
$$

$\Delta T$ can be extracted numerically from:

$$
\frac{d E}{d V}=\rho \int_{T_{0}}^{T_{0}+\Delta T} C_{p}(T) d T
$$

\section{RESULTS AND DISCUSSION}

The maximum depositions, maximum temperature load to graphite and maximum energy flow out of the magnets are summarised in Tables 3 to 5 .

Table 3: Summary of instantaneous load due to asynchronous dump at $7 \mathrm{TeV}$

\begin{tabular}{|l|l|l|l|}
\hline & Peak load J/cm3 & $\Delta \mathbf{T}(\mathbf{K})$ & Energy flow (J) \\
\hline TCDQ & 2139 & 712 & - \\
\hline TCS & 2283 & 679 & - \\
\hline TCDQM & 44.5 & 12.8 & - \\
\hline MCBY & 26.2 & - & 262 \\
\hline MQY & 38.0 & - & 1836 \\
\hline
\end{tabular}

Table 4: Summary of load in one second due to secondary beam halo at $7 \mathrm{TeV}$

\begin{tabular}{|l|l|l|l|}
\hline & Peak load J/cm3 & $\Delta$ T (K) & Energy flow (J) \\
\hline TCDQ & 0.73 & 0.30 & - \\
\hline TCS & 0.59 & 0.25 & - \\
\hline TCDQM & 0.029 & 0.008 & - \\
\hline MCBY & 0.017 & - & 0.154 \\
\hline MQY & 0.024 & - & 0.985 \\
\hline
\end{tabular}

Table 5: Summary of load in one second due to secondary halo at $450 \mathrm{GeV}$

\begin{tabular}{|l|l|l|l|}
\hline & Peak load J/cm3 & $\Delta$ T (K) & Energy flow (J) \\
\hline TCDQ & 0.13 & 0.057 & - \\
\hline TCS & 2.4 & 0.98 & - \\
\hline TCDQM & 0.33 & 0.097 & - \\
\hline MCBY & 0.12 & - & 1.50 \\
\hline MQY & 0.12 & - & 2.33 \\
\hline
\end{tabular}

\section{Asynchronous dump}

The TCDQ was found to absorb most of the energy, Fig. 4. The TCS is exposed to the small number of secondary particles escaping TCDQ, and also two proton bunches that impinge directly on the jaw, creating a highly localised $\sim 800{ }^{\circ} \mathrm{C}$ peak in the temperature profile. The energy deposition in the TCDQ, TCS and TCDQ elements was well below the damage threshold.

Downstream elements are affected only by low energy particles leaving the TCS. Loading to the MQY is greater than that of the more forward MCBY. This effect is attributed to cascade particles that scatter out of the 
upstream volumes, are swept off axis by the MCBY magnetic field and impact on the MQY internal surface.
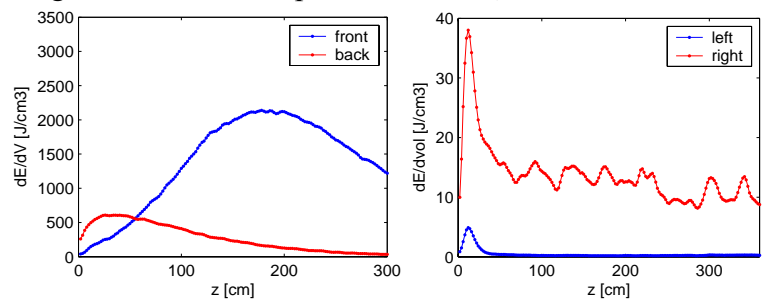

Figure 4: Energy deposition profile along the TCDQ (left) and MQY, for an asynchronous dump.

\section{$450 \mathrm{GeV}$ secondary halo}

At $450 \mathrm{GeV}$ the fraction of protons in the halo beyond $8 \sigma$ is small. Therefore the protection offered by the TCDQ to the TCS right hand jaw has little effect and the energy deposition is essentially symmetrical. Again the protection offered by the TCS means the peak loading to downstream elements are an order of magnitude lower. Loading to the MQY is enhanced by the bending of cascade particles by the MCBY dipole field and impinging on the inner surface of the beam-pipe aperture.

\section{$7 \mathrm{TeV}$ secondary halo}

Loading to the rear TCDQ block is a factor 10 lower than that in the upstream volume. The TCS loading is asymmetrical, the right-hand jaw being largely protected by the TCDQ. Being protected by the TCS, the peak loading to downstream elements is an order of magnitude lower but continues to display left-right asymmetry. The MQY load is a factor of 5 higher than at $450 \mathrm{GeV}$, Fig. 5.
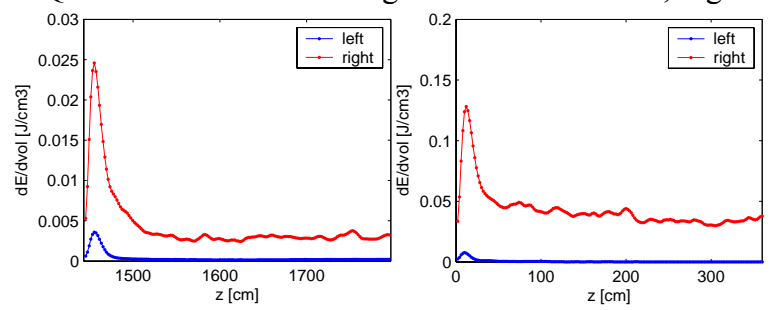

Figure 5: Energy deposition profile along the MQY for $450 \mathrm{GeV}$ (left) and $7 \mathrm{TeV}$ secondary halo load.

\section{IMPLICATIONS}

\section{Protection against asynchronous dump}

The TCS/TCDQ/TCDQM system fulfils its primary design objective of protecting the Q4 magnet, the LHC arc and the LHC inner triplet from destruction in the event of an asynchronous beam dump, up to LHC ultimate intensity. The energy deposition in the Q4 and the protection elements themselves are all within acceptable limits. An important condition that the system operates correctly is that the jaw positions relative to the beam can be maintained to within $0.5 \sigma$.
Prevention of quench from abort gap population in the event of a normal dump

$40 \mathrm{~J} / \mathrm{cm}^{3}$ are deposited in the MQY coil, with ultimate bunch intensity of $1.710^{11} \mathrm{p}+\left(210^{10} \mathrm{p}+/ \mathrm{m}\right)$. Assuming the quench limit is $4 \mathrm{~mJ} / \mathrm{cm}^{3}$, the maximum abort gap population which can be tolerated at $7 \mathrm{TeV}$ corresponds to $1.710^{7} \mathrm{p}+/$ bunch $\left(210^{6} \mathrm{p}+/ \mathrm{m}\right)$. At $450 \mathrm{GeV}$ the corresponding limit in abort gap population will be at least two orders of magnitude higher; limits of $210^{9} \mathrm{p}+/$ bunch $\left(210^{8} \mathrm{p}+/ \mathrm{m}\right)$ are assumed. This is important for specifying both the abort gap monitoring and abort gap cleaning systems currently planned for the LHC.

\section{Prevention of quench from halo particles during low beam lifetime}

The results show that at $450 \mathrm{GeV}$ the maximum power deposited in Q4 is around $120 \mathrm{~mW} / \mathrm{cm}^{3}$, with a total power in the magnet of about $2.3 \mathrm{~W}$. For $7 \mathrm{TeV}$ the corresponding figures are $24 \mathrm{~mW} / \mathrm{cm}^{3}$ and $1 \mathrm{~W}$, respectively. The limit on the total power deposited is assumed to be $34 \mathrm{~W}$ at all energies; here the situation seems comfortable. However, for the localised DC power, the assumed limits are in the range $1-10 \mathrm{~mW} / \mathrm{cm}^{3}$ at $450 \mathrm{GeV}$ and $0.2-5 \mathrm{~mW} / \mathrm{cm}^{3}$ at $7 \mathrm{TeV}$. The simulations with the assumptions detailed above show power depositions a factor of 12-120 and 5-100 higher than these limits, at $450 \mathrm{GeV}$ and $7 \mathrm{TeV}$, respectively. These preliminary results show already that the power deposited in the Q4 magnet during low beam lifetime will be a serious concern for LHC operation.

\section{CONCLUSION AND OUTLOOK}

The TCDQ system performs its protection function adequately; however, there are serious concerns about the secondary halo load at injection and top energy, which might lead to quenches of the downstream Q4. The TCDQM mask design has been redesigned to improve the protection of the Q4 coils, and the model and simulations will be extended and refined, with the new geometry including the Q4 beam screen and cold bore. More accurate halo profiles will be generated by tracking through the full LHC aperture model. The required setting of the TCDQ system will be examined with tracking studies to see if it can be relaxed somewhat. It is hoped that these changes will improve significantly the numbers given above.

\section{REFERENCES}

[1] O. Brüning et al. (eds), "LHC Design Report Volume I - the LHC Main Ring”, CERN-2004-003, 2004.

[2] A.Presland, B.Goddard, "The Performance of the TCDQ diluter System", CERN LHC-Project-Note362, 2005.

[3] FLUKA: Status and Prospective for Hadronic Applications, A. Fassò, A. Ferrari, J. Ranft, P.R. Sala, MonteCarlo 2000, A. Kling, et al. Eds, Springer-Verlag Berlin, p. 955-960 (2001). 